\title{
Efeito de dois agentes tripanosomicidas, violeta de genciana e WR6026, na preservação de concentrado de plaquetas
}

\author{
Effects of two trypanosomicide drugs, gention violet \\ and WR6026, on platelet concentrates preservation
}

\author{
Sheila Soares ${ }^{1}$, Hélio Moraes-Souza ${ }^{1}$, Maria Aparecida Vieira Tostes $^{1}$ e Dayse Maria Lourenço ${ }^{2}$
}

\begin{abstract}
RESUMO
Visando verificar a viabilidade do concentrado de plaquetas, após sua preservação com substâncias, que adicionadas ao sangue sejam capazes de destruir o Trypanosoma cruzi, testamos concentrado de plaquetas estocado com a violeta de genciana e a quinolina WR6026. A viabilidade foi avaliada através da contagem do número de plaquetas, da medida do pH e agregação plaquetária com par de agonistas durante seu período de estocagem. Alterações significativas foram observadas nos concentrados plaquetários tratados com violeta de gen ciana sugerindo comprometimento da viabilidade plaquetária. Com o WR6026 foram observados valores similares aos controles, abrindo perspectivas para 0 emprego deste sal na quimioprofilaxia da doença de Chagas transfusional, em regiões altamente endêmicas.
\end{abstract}

Palavras-chaves: Doença de Chagas transfusional. Concentrados de plaquetas. Quimioprofilaxia. Violeta de genciana. Quinolina WR6026.

\begin{abstract}
In order to verify the viability of platelet concentrate during its preservation period when added with two trypanosomicidal chemical substances, gentian violet and quinoline WR6026, we tested these concentrates and verified the following parameters: number of platelets, $\mathrm{pH}$ and platelet aggregation test with a pair of agonists. Significant alterations were observed in the platelet concentrate treated with gentian violet suggesting important damage to the platelet viability. With WR6026 similar values to the controls were observed, opening new perspectives for the utilization of this salt for the chemoprophylaxis of transfusional Chagas' disease in highly endemic regions.
\end{abstract}

Key-words: Transfusional Chagas'disease . Platelets concentrate. Chemoprophylaxis. Gentian violet. Quinoline WR6026.

A transmissão da doença de Chagas (DC) por transfusão sangǘnea é relatada há várias décadas ${ }^{12}$. Nos dias atuais, com 0 controle da transmissão natural, via erradicação do vetor e as migrações do chagásico para centros urbanos de países endêmicos e não endêmicos, a transmissão transfusional da DC passou a ser 0 principal mecanismo de disseminação da doença ${ }^{9}$. A prevalência média da infecção chagásica entre doadores de sangue na América Latina na década de oitenta era de aproximadamente 7\%, reduzindo para $3 \%$ na década subseqüente. Atualmente este índice, que decresceu sensivelmente em países como Equador $(0,2 \%)$ e Brasil (0,6\%), continua ser de $14,8 \%$ na Bolivia ${ }^{10}$

Nas regiões endêmicas para $\mathrm{DC}$ com transmissão ativa a alta prevalência de sorologia positiva para Trypanosoma cruzi compromete 0 suprimento de sangue e aumenta 0 risco transfusional, pela possibilidade de transfundir hemocomponente com sorologia falso negativa.

Todos estes fatos vêm despertando a comunidade científica para busca de substâncias que adicionadas ao sangue sejam capazes de esterilizá-lo, sem alterar a viabilidade dos seus componentes. As substâncias com este potencial são a violeta de genciana (VG), identificada como eficiente tripanossomicida desde $1953^{15}$, e recentemente a quinolina WR6026 ${ }^{1}$. Moraes-Souza et al ${ }^{11}{ }^{12}$ demonstraram que 0 eritrócito preservado em presença da VG ou do WR6026 mantém sua viabilidade, reforçando o potencial de uso dos mesmos na quimioprofilaxia da DC.

\footnotetext{
1. Faculdade de Medicina do Triângulo Mineiro e Hemocentro Regional de Uberaba, Uberaba, MG. 2. Universidade Federal de São Paulo, São Paulo, SP. Endereço para correspondência: Dra. Sheila Soares. Hemocentro Regional de Uberaba. Av. Getúlio Guaritá 250, Abadia, 38025-440 Uberaba, MG. Telefax: 5534 3312-5077.

e-mail: hemocentro@met.com.br

Recebido para publicação em 16/12/2003

Aceito em 20/3/2004
} 
Com 0 crescente aumento no uso de transfusões de plaquetas em pacientes trombocitopênicos, e 0 emprego quase exclusivo de concentrados individuais de plaquetas, 0 risco de contaminação do receptor pela possibilidade de receber unidades com sorologia falso-negativa tem aumentado consideravelmente nas regiões endêmicas. Portanto, a identificação de drogas com segura ação tripanossomicida, que não acarretem efeito colateral no receptor e nem alterações metabólicas e funcionais sobre plaquetas, é plenamente justificável e é 0 objeto deste estudo.

\section{MATERIAL E MÉTODOS}

Os concentrados de plaquetas ( $\mathrm{CPs}$ ) foram obtidos de doadores aptos, segundo critérios da Portaria número 1376/93, do Ministério da Saúde, e que negassem uso de anti-inflamatórios não hormonais nos últimos 10 dias, anticoncepcionais orais e ingestão de bebidas alcoólicas nos últimos 3 dias.

Foram obtidos CPs pela técnica do plasma rico em plaquetas ${ }^{3}$. Para diminuir a variabilidade da função e do número de plaquetas, quatro unidades de CPs foram agrupadas em pool de $200 \mathrm{ml}$, e novamente divididas em quatro alíquotas: a uma era adicionada VG concentração final 250 $\mathrm{\mu g} / \mathrm{ml}$ ( 1:4000); à outra, VG concentração final 62,5 $\mu \mathrm{g} / \mathrm{ml}$ (1:16.000); e ácido ascórbico na terceira, o WR6026 na concentração final de 2,6 $\mu \mathrm{g} / \mathrm{ml}$ e a última era empregada como controle.

Todos os concentrados plaquetários foram mantidos sob temperatura de $22^{\circ} \mathrm{C}$ e constante rotação em agitador Fisher Scientific $^{\circledR}$, da Kawasumi Laboratories INC a 8rpm.

Amostras dos CPs, aproximadamente $3 \mathrm{ml}$, foram obtidas das respectivas bolsas nos tempos: 3; 24; 48 e 72 horas, após 0 processamento do sangue e submetidas às seguintes análises:

Contagem plaquetária. Foi realizada em $10 \mathrm{CPs}$, por método eletrônico, empregando-se 0 contador automático de células Coulter T890 ${ }^{\circledR}$ (Coulter Electronics, Inc, Hialeach, F, USA). Para a obtenção do número de plaquetas do CP, empregou-se a seguinte fórmula:

№ Plaq. Bolsa $=$ № Plaq. amostra $/ \mathrm{mm}^{3} \times 1000 \times$ Volume do CP.

Medida do pH. Foi realizado em $15 \mathrm{CPs}$, usando um determinador de pH marca Mettler Toledo ${ }^{\circledR} 320$.

Agregaçáo plaquetária. Foi realizada em $10 \mathrm{CPs}$, utilizando-se 0 agregômetro Chrono-log Corporationâ, modelo 500-VS. 0 método utilizado foi 0 de Born (1963), sendo empregados os seguintes pares de agonistas: ácido aracdônico $(5,5 \mu \mathrm{mol})$ e adrenalina $(1,25 \mu \mathrm{mol})$ e ácido aracdônico e adenosina disfosfato (ADP 2,5 $\mu \mathrm{mol}$ ). A agregação espontânea foi avaliada após ou durante três minutos de registro, sem adição de agonistas. Os resultados foram expressos em percentagem de agregação.

Métodos estatísticos. Para analisar a variação de um determinado parâmetro, ao longo do tempo de estocagem (de 3 a 72 horas), foi empregado o Teste de Friedman e, para comparar as médias obtidas em cada tempo, nos quatro diferentes grupos estudados, foi usado o teste ANOVA. Em todos os testes foi fixado em 0.05 ou $5 \%$ o nível para a rejeição da hipótese de nulidade.

\section{RESULTAD0S}

Contagem de plaquetas. Amédia do número de plaquetas, obtidas nos diferentes tempos e com diferentes drogas e/ou concentrações destas, está representada na Tabela 1.

Tabela 1- Média do número de plaquetas (x $10^{10}$ ) em CP tratados com VG 1:4000, VG 1:16.000, WR6025 e controle, nos diferentes tempos de estocagem.

\begin{tabular}{lccccr}
\hline Tempo & Controle & VG1:4000 & VG 1:16000 & WR & ANOVA \\
\hline 3h & $5,6 \pm 0,4$ & $5,5 \pm 0,4$ & $5,5 \pm 0,5$ & $5,8 \pm 0,5$ & $\mathrm{P}>0,05$ \\
24h & $5,3 \pm 0,3$ & $5,0 \pm 0,3$ & $5,1 \pm 0,5$ & $5,3 \pm 0,3$ & $\mathrm{P}>0,05$ \\
$48 \mathrm{~h}$ & $5,3 \pm 0,3$ & $5,0 \pm 0,4$ & $4,9 \pm 0,6 *$ & $5,2 \pm 0,3$ & $\mathrm{P}=0,0001$ \\
$72 \mathrm{~h}$ & $5,1 \pm 0,3$ & $4,4 \pm 0,4 *$ & $4,6 \pm 0,6$ & $5,1 \pm 0,4$ & $\mathrm{P}=0,0001$ \\
\hline Friedman & $\mathrm{P}=0,0001$ & $\mathrm{P}=0,0001$ & $\mathrm{P}=0,0001$ & $\mathrm{P}=0,0001$ &
\end{tabular}

* diferença observada na ANOVA.

Houve redução estatisticamente significante do número de plaquetas em todos os grupos durante a estocagem (Friedman $\mathrm{p}=0,001$ ), conforme mostra a Tabela 1 .

Na comparação entre os diferentes grupos, CPs tratados com violeta de genciana apresentaram maiores quedas com significância estatística às 48 horas com a VG16 e às 72 horas com a VG 1:4000 (Figura 1).

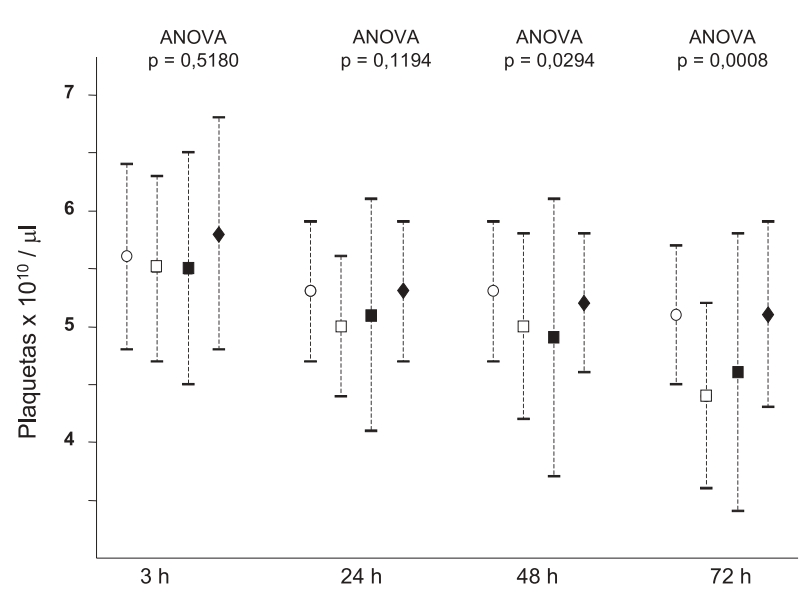

Figura 1 - Contagem de plaquetas em diferentes tempos nos CPs tratados com VG 1:4000(口), VG1:16.000( $)$, WR 6026( ) e controle (O) e valor de "p" obtido após a aplicação do teste de ANOVA.

Medida do pH. Aumento em quase todos os CPs ao longo da estocagem foi observado, sendo este estatisticamente significante (Friedman $\mathrm{p}=0,0001$ ) em todos, exceto no $\mathrm{CP}$ com VG1: 16.000, nos quais houve redução significante, como está mostrado na Tabela 2 e Figura 2.

Agregação plaquetária. Não houve agregação espontânea em nenhum CP ao longo do período de preservação 
Tabela 2 - Média dos valores do pH nos CPs estocados com VG1:4000, VG 1:16.000, WR 6026 e controles.

\begin{tabular}{lccccr}
\hline & Controle & VG1:4000 & VG1:16000 & WR & ANOVA \\
\hline $3 \mathrm{~h}$ & $7,17 \pm 0,14$ & $7,13 \pm 0,09$ & $7,13 \pm 0,09$ & $7,14 \pm 0,10$ & $\mathrm{P}>0,05$ \\
$24 \mathrm{~h}$ & $7,25 \pm 0,12$ & $7,18 \pm 0,12$ & $7,02 \pm 0,22 *$ & $7,30 \pm 0,08$ & $\mathrm{P}=0,0001$ \\
$48 \mathrm{~h}$ & $7,34 \pm 0,15$ & $7,27 \pm 0,15$ & $7,01 \pm 0,31 *$ & $7,32 \pm 0,19$ & $\mathrm{P}=0,0001$ \\
$72 \mathrm{~h}$ & $7,43 \pm 0,21$ & $7,41 \pm 0,19$ & $7,00 \pm 0,41 *$ & $7,34 \pm 0,41$ & $\mathrm{P}=0,0001$ \\
\hline Friedman & $\mathrm{P}=0,0001$ & $\mathrm{P}=0,0001$ & $\mathrm{P}=0,4307$ & $\mathrm{P}=0,0001$ & \\
\hline * difo
\end{tabular}

* diferença observada na ANOVA.

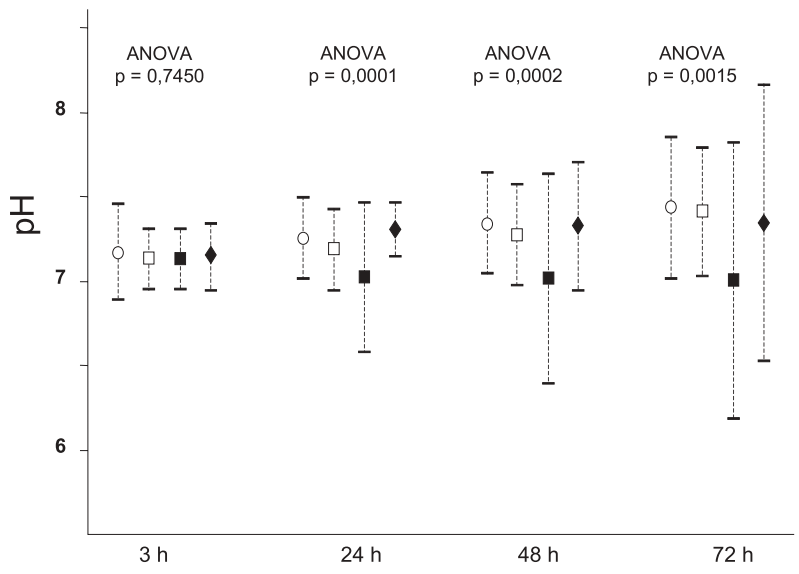

Figura 2 - Medida do $\mathrm{pH}$ em diferentes tempos para o controle $(\mathrm{O})$ e os CPs tratados com VG 1:4000 (口), VG1:16.000(口) e WR 6026 $(\bullet)$ e 0 valor de "p" obtido após a aplicação do teste de ANOVA.

( dados não mostrados) . A agregação plaquetária induzida por pares de agonistas mostrou-se reduzida desde a terceira hora em todos os CPs. Para as duas concentrações de VG, porém, redução estatisticamente significante foi observada apenas nos controles e com a VG-1:4000, com pares de agonistas AA+ADP (Tabelas 3 e 4).

Na comparação entre os diferentes grupos, observa-se queda mais acentuada nos concentrados tratados com VG. Nas duas concentrações, porém, diferença significante estatisticamente foi verificada apenas às 24 horas, nas amostras com VG 1:4000 e com os pares de agonistas AA+ADP ( $p=0,001)$ (Tabelas 3 e 4).

Tabela 3 - Média dos valores de agregação plaquetária dos CPs tratados com VG- 1:4000, VG- 1:16000, WR 6026 e controles, induzida pelos pares de agonistas AA + Adrenalina, nos diferentes tempos de estocagem.

\begin{tabular}{lccccc}
\hline Tempos & Controle & VG1:4000 & VG1:16000 & WR & ANOVA \\
\hline 3h & $74 \pm 19$ & $56 \pm 23$ & $59 \pm 08$ & $76 \pm 10$ & $\mathrm{P}>0,05$ \\
24h & $61 \pm 20$ & $41 \pm 18^{*}$ & $51 \pm 20$ & $70 \pm 14$ & $\mathrm{P}=0,0001$ \\
48h & $57 \pm 29$ & $37 \pm 13$ & $47 \pm 20$ & $60 \pm 23$ & $\mathrm{P}>0,05$ \\
72h & $47 \pm 20$ & $37 \pm 22$ & $47 \pm 22$ & $47 \pm 29$ & $\mathrm{P}>0,05$ \\
\hline Friedman & $\mathrm{P}=0,0039$ & $\mathrm{P}=0,0161$ & $\mathrm{P}=0,1402$ & $\mathrm{P}=0,0867$ &
\end{tabular}

Tabela 4 - Média dos valores de agregação plaquetária dos CPs tratados com VG- 1:4000, VG- 1:16000, WR 6026 e controles, induzida pelos pares de agonistas $\mathrm{AA}+\mathrm{ADP}$, nos diferentes tempos de estocagem.

\begin{tabular}{lccccc}
\hline Tempos & Controle & VG1:4000 & VG1:16000 & WR & ANOVA \\
\hline 3h & $63 \pm 20$ & $51 \pm 15$ & $48 \pm 14$ & $64 \pm 15$ & $\mathrm{P}>0,05$ \\
24h & $53 \pm 20$ & $44 \pm 10$ & $46 \pm 14$ & $63 \pm 15$ & $\mathrm{P}>0,05$ \\
48h & $48 \pm 22$ & $44 \pm 14$ & $40 \pm 20$ & $53 \pm 19$ & $\mathrm{P}>0,05$ \\
$72 \mathrm{~h}$ & $43 \pm 19$ & $38 \pm 13$ & $41 \mathrm{v} 16$ & $40 \pm 27$ & $\mathrm{P}>0,05$ \\
\hline Friedeman & $\mathrm{P}=0,2933$ & $\mathrm{P}=0,3296$ & $\mathrm{P}=0,7692$ & $\mathrm{P}=0,1176$ &
\end{tabular}

*Diferença observada na ANOVA.

\section{DISCUSSÃ0}

A diminuição do número de plaquetas, durante 0 período de estocagem referida na literatura ${ }^{4}{ }^{6}$, é explicada tanto pela formação de microagregados plaquetários como pela lise das plaquetas. Em nosso material, esta queda foi significante em todos os grupos estudados, com exceção dos valores médios obtidos às 3 horas de estocagem, quando todos estavam abaixo dos limites mínimos, de acordo com os critérios da Associação Americana de Bancos de Sangue. Estes valores podem ser explicados pelo método empregado na contagem e/ou pela redução do volume dos CPs pela retirada de amostras para a realização dos testes ${ }^{8}$. Assim é que estudos têm demonstrado que a contagem eletrônica geralmente registra valores 20\% inferiores à contagem microscópica e que a redução do volume, abaixo dos 50ml, interfere na viabilidade das plaquetas, podendo até provocar sua lise?

Ao compararmos o número de plaquetas dos $\mathrm{CPs}$ expostos às diversas drogas com 0 controle, foi observado que às 48 horas e às 72 horas a diminuição foi significante naqueles que continham VG1.16:000 e 1:4.000, respectivamente. Tais achados sugerem que a VG, mesmo em pequenas concentrações, interferiu na preservação plaquetária, possivelmente induzindo a formação de grumos ou lise. Na verdade, a inspeção microscópica dos esfregaços do material obtido dos CPs tratados com VG evidenciou a presença des agregados plaquetários, 0 que não foi observado com os controles e com as amostras tratados com WR6026.

Quando ao pH, existe preocupação com a acidificação do meio durante a estocagem, 0 que interfere na viabilidade das plaquetas ${ }^{4}$. No presente estudo observamos, ao contrário, elevação estatisticamente significante do pH durante a estocagem nos CP controle e naqueles tratados com WR6026 e com VG 1:4000, apesar de todos terem se mantido dentro dos limites normais. Portanto, 0 uso destes agentes tripanossomicidas parece não alterar o pH dos CPs de modo a comprometer a viabilidade das plaquetas estocadas.

0 estudo da agregação plaquetária nos CPs é realizado para avaliar a capacidade funcional das plaquetas estocadas ${ }^{14}$. Várias são as dificuldades desta avaliação, uma vez que a agregação plaquetária in vitro com agentes agonistas isolados não reflete a qualidade hemostática das plaquetas estocadas ${ }^{13}$.

A divergência entre a baixa agregação plaquetária in vitro com agente agonista único e a adequada resposta hemostática in vivo pode ser explicada quando se utiliza dois agentes agonistas simultaneamente e observa-se taxas de agregação satisfatórias².

A agregação plaquetária com os pares de agonistas empregados no presente estudo evidenciou, sobretudo nos CPs controles e com WR6026, valores comparáveis aos referidos na literatura ${ }^{2} .0$ encontro de níveis de agregação iguais ou superiores aos controles nos PCs preservados com WR6026 sugere que este sal não interfere na viabilidade plaquetária. Já os CPs tratados com VG 1:4.000 e 1:16.000 apresentaram taxa de agregação reduzida em relação ao controle, em todos os momentos de preservação, sugerindo 
que a VG interfere na agregação e, possivelmente, na função de plaquetas preservadas.

Tomados em conjunto, os resultados deste estudo sugerem que 0 uso de VG como quimioprofilático da DC transfusional não é adequado para 0 tratamento de $\mathrm{CP}_{\mathrm{S}}$. 0 efeito deletério da VG sobre as plaquetas foi demonstrado pela formação de microagregados à microscopia e na redução da intensidade da agregação plaquetária.

Por outro lado, o uso do WR6026 parece promissor no tratamento quimioprofilático dos CPs. Não se observou alteração da contagem de plaquetas, dos níveis de $\mathrm{pH}$ e nem da agregação plaquetária induzida por par de agonistas, 0 que nos permite sugerir ser este agente um forte candidato a substituir a VG na quimioprofilaxia da DC transfusional, especialmente em regiões com altas prevalências de doadores chagásicos e/ou que não disponham de sorologia segura. Esta droga, além de incolor, o que minimiza a rejeição dos transfusionistas, (rejeição freqüentemente demonstrada à VG), se revelou relativamente pouca tóxica quando empregada no tratamento da leishmaniose viscera ${ }^{16}$.

No entanto, ensaios clínicos e avaliação da lesão de estocagem, como a medida da P-selectina ${ }^{5}$, seriam recomendáveis antes do seu emprego sistemático no sangue e ou hemocomponentes estocado para fins transfusionais.

\section{REFERÊNCIAS BIBLIOGRÁFICAS}

1. Chiari E, Oliveira B, Prado MF, Alves RJ, Galvão LMC, Araujo FG. Potential use of WR6026 as prophylaxis against transfusion-transmitted American trypanosomiasis. Antimicrobial Agents and Chemotherapy 40: 613-615, 1996.

2. Di Minno G, Silver MJ, Murphy S. Stored human platelets retain full aggregation potential in response to pairs of aggregating agents. Blood 59:563-567, 1982
3. Givisiez, F Netal. Preparação de Hemocomponentes. Ministério da Saúde, Brasília, DF, p.91,1999.

4. Holme S. Storage and quality assessment of platelets. Vox Sanguinis 74, ( supl 2) : 207-216, 1998.

5. Holme S, Sweeney JD, Sawyer S, Elfath MD. The expression of P-selectin during collection, processing, and storage of platelet concentrates: Relationship to loss of in vivo viability. Transfusion 37:12-17, 1997.

6. Klinger MHF. The storage lesion of platelets: Ultrastructural and functional aspects. Annals of Hematology 73:103-112, 1996.

7. Krishnan LK, Sulochana PV, Mathai J, John A, Sivakumar R. Morphological and ultrastructural changes of platelet concentrates stored in PVC bags. The Indian Journal of Medical Research 105:77-84,1997.

8. Lippa S, Mores N, Aureli V, Fagiolo E. Biochemical and Functional Changes of Platelets Stored for Transfusional Use. Folia Haematologica 114:680-685, 1987.

9. Moraes-Souza H. Transmissão transfusional da doença de Chagas. Revista de Patologia Tropical 29 ( supl) : 91-100,2000.

10. Moraes-Souza H, Bordin JO. Strategies for prevention of Transfusion Associated Chagas' Disease. Transfusion Medicine Reviews 10: 1-11,1996.

11. Moraes-Souza H, Kerbauy J, Barreto OCOP, Puhler GMP, Nonoyama K, Juliano Y. Metabolism and preservation of fresh and stored erythrocytes in blood treated with gentian violet. Brazilian Journal Medicine Biological Research 21:241-246, 1988.

12. Moraes-Souza H, Pianetti GM, Barreto OCO, Nonoyama K, Grolg M, Chiari E. The aminoquinoline WR6026 as a probable substitute of gentian violet for the Chagas' Disease prophylaxis in preserved blood for transfusion. Transfusion 37 ( supl.) : 135s,1997.

13. Moroff G. Aggregation: release response of platelets stored at a $22^{\circ}$. Vox Sanguinis 40 ( supl1):110-114,1981.

14. Murphy S, Gardner FH. Platelet Storage at $22^{\circ} \mathrm{C}$; Metabolic, Morphologic, and Functional Studies. The Journal of Clinical Investigation 50:370-376, 1971.

15. Nussenzweig V, Biancalana A, Amato Neto V, Sonntag R, Freitas JLP De and Kloentzel J. Nota Prévia - Ação da violeta de genciana sobre o Tripanosoma cruzi in vitro: Sua importância na esterilização do sangue destinado à transfusão. Revista Paulista de Medicina 42:57-58, 1953.

16. Sherwood JÁ, Gachihi S, Muigai RK, Skillman DR, Mugo M, Rashid JR, Wasunna KMA, Were JBO, Kasili SK, Mbugua JM, Kirigi G, Schaefer KU, Oster CN, Fleckenstein LL, Berman JD, Brewer TG, Roberts CR, Johnson AJ, and Schuster BG. Phase 2 Efficacy Trial of an Oral 8-Aminoquinoline (WR6026) for Treatment of Visceral Leishmaniasis. Clinical. Infections Diseases 19:1034-9,1994. 